\title{
Reformas digitales en TVN. Cambios hacia un servicio público más integral
}

\section{Digital Reforms in Chilean National Television: Towards a More Comprehensive Public Service}

\author{
Valerio Fuenzalida \\ Pontificia Universidad Católica de Chile \\ vfuenzal@vtr.net
}

\begin{abstract}
Resumen
Chile finalmente adoptó la norma nipo-brasileña para las emisiones en TV digital abierta o terrestre (14 septiembre 2009). Esta tecnología permitirá que TVN en su mismo canal físico de $6 \mathrm{Mhz}$ pueda emitir dos señales en alta definición (HD) y hasta 7 señales en definición estándar.

La tecnología digital tiene una importancia estratégica para repensar la "misión" televisiva de TVN, canal público chileno, y para las tareas del CNTV, agencia reguladora de la TV. Y la tecnología digital ofrece una oportunidad para ampliar y complejizar el servicio público que TVN puede ofrecer a la audiencia chilena, en particular frente a los importantes cambios en Canal 13 y CHV.
\end{abstract}

Palabras clave: TVN, televisión pública, televisión digital, televisión chilena.

\begin{abstract}
Chile finally adopted Japanese-Brazilian standard for digital TV open broadcasts (September 14th, 2009). This technology will allow Chilean National Television (TVN), in the same physical channel of $6 \mathrm{MHz}$, emit two signals in High Definition (HD) and up to 7 standard definition signals.

Digital technology is strategically important to think the "mission" of TVN, the Chilean public channel, as same as the tasks of the Chilean National Council for Television. Digital technology also offers an opportunity to expand and complicate the public service TVN can offer to Chilean audience, particularly in face to significant changes in its competitors Channel 13 and ChileVision.
\end{abstract}

Keywords: TVN, Public television, Digital television, Chilean television. 


\section{La reforma de TVN en el año 1992}

La reforma legal del año 1992 asignó a TVN una misión minimalista de proveer una información plural, balanceada, confiable, y masiva a la sociedad chilena.

La situación de la información televisiva al final de la dictadura era que el rating promedio total anual en el año 1989 a los noticiarios centrales señalaba 30,0 puntos para Teletrece (de Canal 13) y menos de la mitad (13,7 puntos) para 60 Minutos de TVN.

El rediseño de los servicios informativos de TVN bajo el nombre de 24 Horas y con el mandato legal de información plural se ha mantenido mantenido por años (y bajo muchas presiones contrarias) y ha dado fruto de credibilidad, como se puede advertir en las cifras de aumento del rating, y finalmente primacía en la competencia con otros informativos centrales.

Tabla 1. Evolución del rating total anual de los noticiarios centrales (fuente: Time-Ibope)

\begin{tabular}{|c|c|c|c|c|}
\hline Año & TVN & Canal 13 & Mega & $\mathrm{CHV}$ \\
\hline 1996 & 18,3 & 20,7 & 7,8 & 2,5 \\
\hline 1997 & 19,7 & 22,3 & 7,3 & 3,3 \\
\hline 1998 & 21,8 & 22,1 & 11,1 & 5,1 \\
\hline 1999 & 22,3 & 19,5 & 10,6 & 6,9 \\
\hline 2000 & 24,7 & 19,8 & 8,8 & 6,7 \\
\hline 2001 & 26,9 & 17,0 & 9,0 & 7,9 \\
\hline 2002 & 23,0 & 14,9 & 14,8 & 9,3 \\
\hline 2003 & 20,6 & 22,5 & 11,6 & 9,5 \\
\hline 2004 & 19,5 & 18,4 & 14,4 & 11,1 \\
\hline 2005 & 15,8 & 21,3 & 12,0 & 11,3 \\
\hline 2006 & 18,1 & 16,0 & 11,2 & 12,6 \\
\hline 2007 & 17,9 & 14,9 & 11,0 & 14,2 \\
\hline 2008 & 16,1 & 14,1 & 11,0 & 14,1 \\
\hline 2009 & 16,4 & 12,3 & 10,3 & 13,9 \\
\hline
\end{tabular}

Otro indicador es el juicio cualitativo acerca del posicionamiento informativo de TVN, en comparación con otros medios. La siguiente tabla constituye un mapa de percepción cualitativa acerca de la ubicación de diversos medios en un continuum entre izquierda- 
derecha. La ubicación 0 indica izquierda y 10 marca derecha. TVN se ubica en el centro, equidistante de ambos polos; esto se interpreta como una percepción de balance, sin marcado sesgo, y una confirmación que la opinión ciudadana ha reconocido la línea editorial plural de TVN. El concepto de objetividad está en decadencia en el mundo académico, porque objetividad significa conocer la verdad desde una postura omnisciente y sin intereses, pero en política más bien hay puntos de vista y opiniones legítimamente diversos. La obligación legal de TVN es entregar balanceadamente los diversos puntos de vista de los actores políticos presentes en la institucionalidad política chilena.

Gráfico 1. Evaluación de medios de comunicación en la escala izquierda (0)-derecha (10) (fuente: Mori-CSES, junio de 2004)

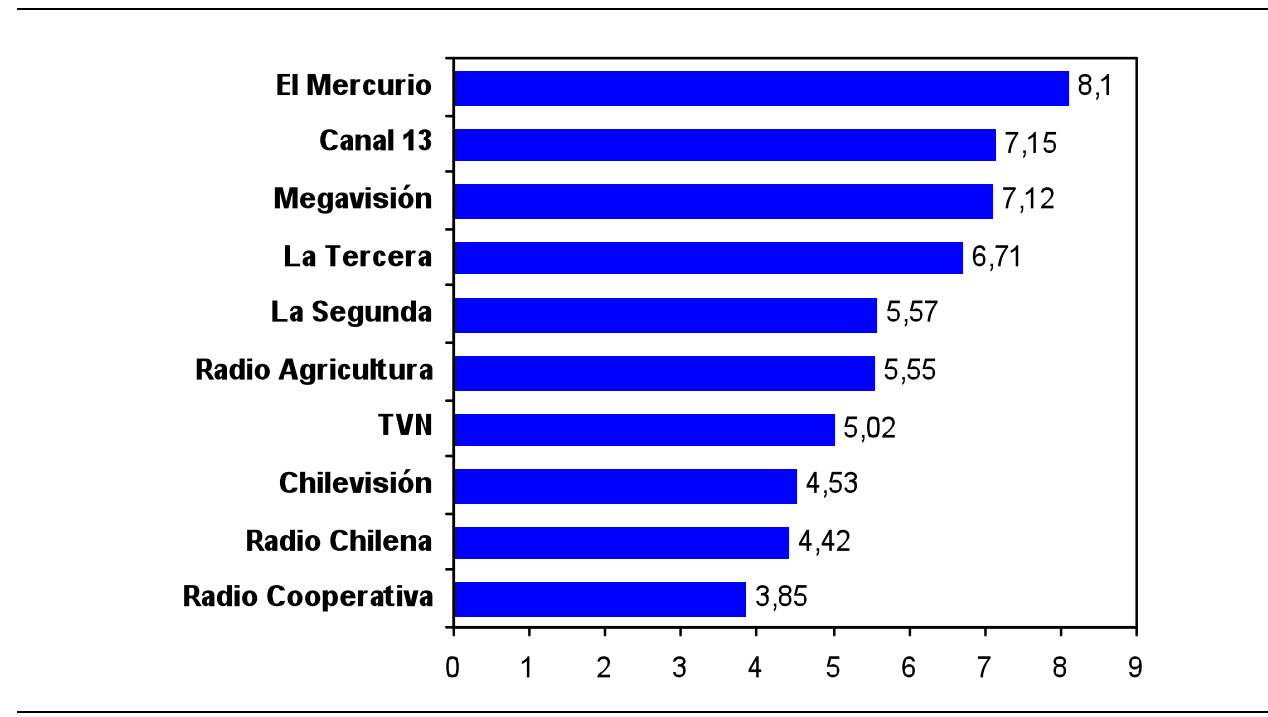

La Tercera Encuesta Nacional de Opinión Pública en el año 2007 de la Universidad Diego Portales muestra que TVN es el canal que se ve con más frecuencia para informarse, con un $37,5 \%$, frente al segundo canal que muestra un 22,9\%. En la encuesta ICSO-UDP de 2009, TVN asciende a un $45 \%$ como el primer canal más visto por los chilenos para informarse; el segundo canal para informarse se mantiene en un $22 \%$.

En el mes de agosto de 2010, apareció un interesante juicio acerca de la reputación corporativa de algunas organizaciones, específicamente por su comportamiento ante el terremoto. La medición efectuada por Hill \& Knowlton Captiva, La Tercera y Collect GFK quiso obtener una imagen del desempeño de empresas ante el terremoto de febrero de 2010: aparece Soprole evaluada en primer lugar con nota 6,2 en escala de 1 a 7, y luego en segundo lugar (junto a otras empresas) TVN, con nota 5,8. Todas las otras empresas evaluadas en segundo lugar son empresas donantes de materiales y alimentos; TVN es la única empresa en el grupo de las mejor evaluada en tanto medio de comunicación, por su labor informativa ante el terremoto. También aparecen bien evaluados Canal 13 (nota 5,6), luego Mega (5,5) y 
CHV (5,4) (La Tercera-Negocios, 22/08/ 2010, pp. 8-9). La reputación corporativa de TVN es alta para la ciudadanía, no solo en credibilidad sino en oportunidad informativa.

La reforma estableció para TVN un Directorio con amplios poderes para ejecutar la misión de proporcionar información pluraly balanceada; tal mecanismo, ha imposibilitado el uso propagandístico del canal, ha desestimulado la demagogia populista y el enfrentamiento autodestructivo; es decir, TVN ha contribuido finalmente a la estabilidad y a la gobernabilidad en el país, a la discusión más racional en los conflictos, a mantener el crecimiento económico, y a enfrentar mejor las recesiones económicas. El Banco Mundial ha señalado en julio del 2007 la relación estadísticamente positiva entre gobernabilidad y crecimiento económico, crecimiento sin el cual es imposible enfrentar seriamente el problema de la pobreza.

Las evaluaciones, externas a la empresa (y más distantes del juicio político con el apasionamiento inmediato) señalan, pues, que tal misión se está cumpliendo (al menos substantivamente) a juicio de la audiencia, quien ha otorgado a la estación pública credibilidad y sintonía. Desde un punto de vista de contenidos, la sección política "La Entrevista del Domingo" en el noticiario central dominical ha fijado para la TV chilena un standard de balance y profundidad (siempre perfeccionables); el hecho que a menudo las opiniones vertidas en esas entrevistas aparezcan en la prensa del día lunes es una confirmación del peso editorial.

No está demás recordar que el proyecto Aylwin-Navarrete para TVN sufrió fuertes críticas desde la propia Concertación, de parte de algunos grupos que querían utilizar la pantalla para sus revanchas político-ideológicas, para intereses partidistas, e incluso intereses personales; pero también recibió críticas de profesionales que esperaban recompensas por su duro combate contra la dictadura. Esas críticas del pasado reaparecen periódicamente en especial cuando el oficialismo o el Poder Ejecutivo se sienten acosados; críticas que a menudo se hacen en voz baja ya que son impresentables públicamente. El proyecto Aylwin-Navarrete tuvo, en cambio, un énfasis ético-político, que se calculaba sería más adecuado para la gobernabilidad, y el desarrollo socio-económico del país. Era una opción de política televisiva más pensada para influir en el mediano y largo plazo ${ }^{1}$.

Esta opción por un pluralismo al interior del canal público se contrapone a la opción del pluralismo entre varios canales competitivos con diversos énfasis ideológicos. La argumentación inicial contra esta segunda opción se basaba en la escasez de frecuencias y en

\footnotetext{
${ }^{1}$ Esta concepción de una influencia masiva, pero minimalista para impedir la desinformación, ha sido comparada a las nuevas concepciones acerca de la misión básica asignada a un Banco Central, cual es mantener el valor de la moneda e impedir la degradación de su valor por la inflación, manipulada políticamente para obtener efectos electorales de corto plazo; del mismo modo, el rol de una información plural y balanceada es impedir la degradación del lenguaje político para abordar las temáticas ciudadanas; degradación posible a través de la propaganda gubernamental, la desinformación manipuladora, los interés ideológicos, las RR PP políticas, el insulto a las posturas discrepantes, las promesas populistas con soluciones ilusorias, etc.; degradación siempre amenazante, especialmente en tiempos electorales, y en tiempos de incertidumbre o debilidad del oficialismo gobernante. En los años recientes, con el recambio en la coalición gobernante de Chile, la Concertación ha reapreciado la misión informacional plural y la estructura jurídica de TVN.
} 
el costo de operación de un canal. Más recientemente hay evidencias que en ambientes informativos de competencia ideológica, y sin un medio plural y masivo, la expresión política tiende a la confrontación polarizada, generando un escenario de consumo selectivo por parte de los receptores y sin diálogo interaccional entre los actores políticos (lyengar y Hahn, 2009). La expresión político-cultural plural y balanceada buscada para TVN pretende no solo la legítima expresión de la competencia política sino además generar un espacio comunicacional de interacción al interior de los actores políticos, espacio de diálogo que se considera esencial para la gobernabilidad democrática.

\section{Demanda insatisfecha por diversidad en la programación}

Según la Sexta Encuesta Nacional de TV realizada por elCNTV (Televisión abierta: Niveles de Satisfacción y Percepción de Calidad; 2008) los medios mejor evaluados son radio y TV cable/satelital, es decir, aquellos que ofrecen más alternativas programáticas, ya que hay una mayor abundancia de estaciones radiales y de canales. Los medios peor evaluados son la TV abierta y diarios, es decir, aquellos donde la diversidad es muy restringida en Chile.

Esa mayor diversidad de canales y en programaciones temáticas explicaría la evolución al alza - en un lapso de diez años - en el nivel de satisfacción frente a la TV cable/satelital frente al decrecimiento en satisfacción que afecta a la TV abierta. En efecto, la TV abierta alcanzaba un 55,6\% de satisfacción en 1999 pero baja ahora a un 46,1\% de satisfacción por parte de la audiencia; en cambio, la TV por cable sube a un 69,2\% de satisfacción.

Talinsatisfacción es la que se constata en elfuerte crecimiento de los hogares abonados al sistema del cable.

Gráfico 2. Evolución del rating por hogar anual, por canal y TV de pago (fuente: Time Ibope)

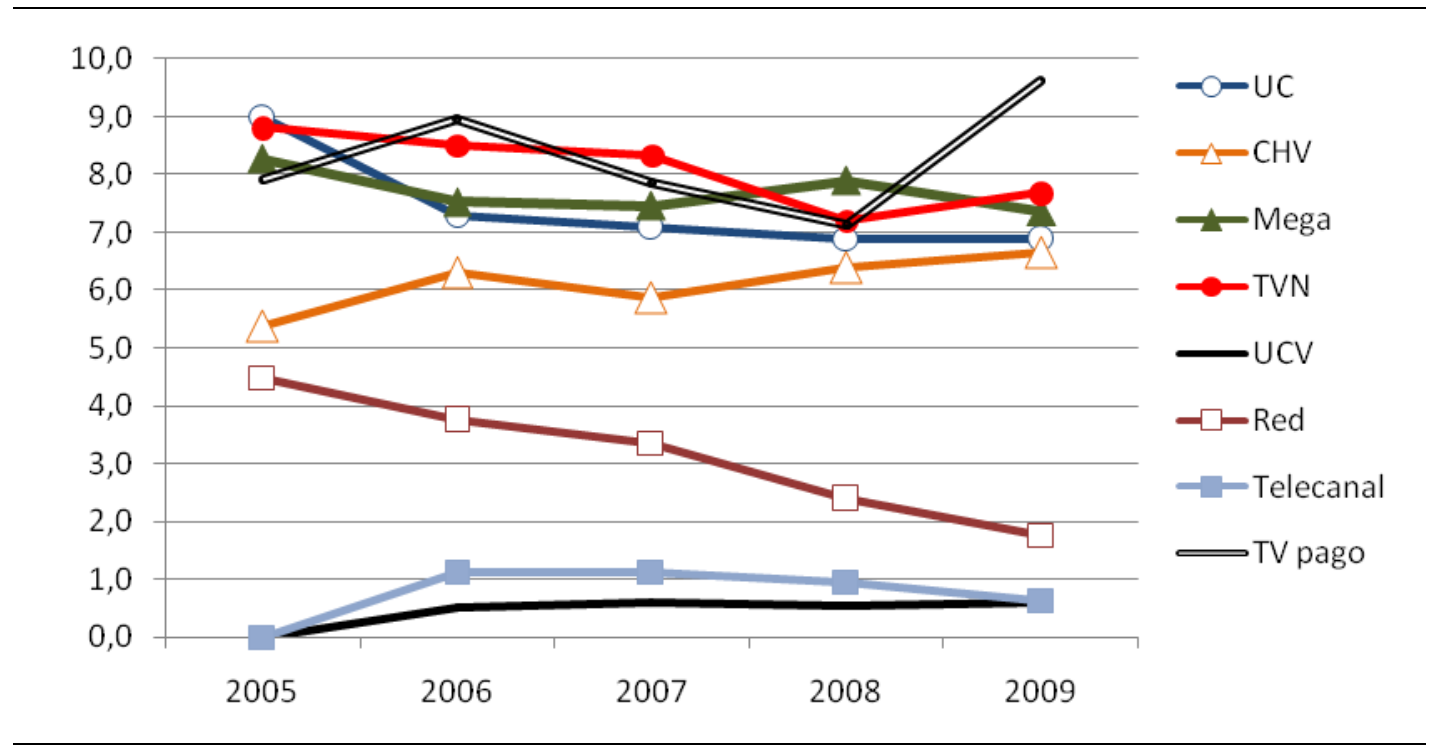


Los datos históricos muestran que TVN se mantiene en primer lugar con un 7,7 de rating promedio anual en el año 2009. En segundo lugar con 7,4 se ubica la estación privada Mega, que el año anterior se ubicó en primer lugar. Canal 13 ha bajado al tercer lugar con 6,9 y muestra un importante descenso en el quinquenio. En cuarto lugar y a corta distancia CHV con 6,6 puntos, estación que muestra una tendencia constante hacia el crecimiento.

Un dato histórico importante es el crecimiento en audiencia del cable, que en conjunto con 9,6 puntos supera el promedio anual del primer canal abierto. Este es un dato que solo se registra con tanta distancia en el año 2009. El año 2009, la TV de pago tenía en todo el país 1.588.867 de subscriptores, que representan un 35\% de penetración en hogares; los datos más actualizados elevan la penetración al $39 \%$.

La interpretación de estos datos cuantitativos y cualitativos permite concluir que la audiencia está reclamando más diversidad programática a la TV abierta chilena ${ }^{2}$. La insatisfacción general con toda la TV abierta chilena permite concluir que la actual demanda social hacia el sistema televisivo -y hacia TVN en particular- es diferente a la demanda por credibilidad, en elinicio de los 90.

\section{Nueva etapa para TVN}

\section{Opción por la diversidad}

Una mayor diversidad programática puede ser satisfecha de modo muy limitado por un canal que emite 20-24 horas diarias con una sola señal generalista.

La TV digital terrestre inaugura una nueva etapa estratégica para la misión pública de TVN, etapa en la cual no solo asegure la información plural, confiable y masiva sino mayor calidad para la audiencia con una oferta televisiva diversificada. El nuevo escenario chileno de canales privatizados debería impulsar en dirección hacia esta diversidad, culturalmente necesaria.

TVN tiene la opción técnica de transformarse en empresa multioperadora ofreciendo varias señales segmentadas con nuevas programaciones temáticas para la audiencia ${ }^{3}$. La operación de diversas señales por el mismo operador posibilita diversidad en la oferta, sumar más audiencia interesada en contenidos diferentes, y baja en los costos; es el modelo de negocios no solo de la BBC en TV digital abierta sino de las programadoras en el cable que ofrecen paquetes de señales segmentadas (p.e. Discovery Channel, Discovery Kids, Discovery Home \& Health, Animal Planet, etc.).

\footnotetext{
2 Adicionalmente, existe también evidencia en otros países acerca del desplazamiento de las audiencias desde las televisiones generalistas hacia canales temáticos, cuando están disponibles en TV abierta.

${ }^{3}$ El debate acerca de TVN en el Parlamento debería efectuarse en permanente relación con el Directorio de TVN; la práctica de la BBC de discutir con la Cámara de los Comunes sus nuevas metas ha sido fundamental para la renovación exitosa de su misión.
} 
Si TVN se convierte en operador multicanal, puede manejar varias señales digitales de cobertura nacional, abiertas a todo el público, y segmentadas con contenidos diversificados, como:

- señal generalista de compañía y ficción

- señal infantily juvenil

- noticias e información socio-política

- señal de alta cultura y ciencia

- señales regionales

En otros textos se ha argumentado ampliamente para destacar la importancia de una señal televisiva pública segmentada hacia niños y en horarios tardíos hacia jóvenes; ante la carencia de programación infantil dedicada, los datos de rating muestran que los niños en Chile y en América Latina se trasladan crecientemente al cable infantil (Fuenzalida, 2008). Una señal de esta naturaleza debe ser trabajada en alianza con la JUNJI y el Ministerio de Educación, quienes pueden entregar también algunos aportes económicos.

Tampoco es necesario justificar largamente la necesidad de un canal segmentado en contenidos culturales, el cual será ciertamente minoritario en audiencia, pero puede entregar contenidos altamente valorados a audiencias que no tendrían acceso sin la TV abierta. Es el canal en donde debería tener un sitio destacado la creciente producción chilena de documentales.

Tabla 2. Síntesis comparativa en categorías generales de programación: emisión y audiencia, 2008 y 2009 (fuente: Time Ibope y Orbitel Chile)

\begin{tabular}{lcccc}
\hline & \multicolumn{2}{c}{ Programación 2009} & \multicolumn{2}{c}{ Programación 2008} \\
\hline Categoría & $\%$ tiempo & $\%$ audiencia & $\%$ tiempo & $\%$ audiencia \\
\hline Ficción & $36.9 \%$ & $34.9 \%$ & $35.1 \%$ & $37.3 \%$ \\
Información & $\mathbf{1 7 . 9 \%}$ & $\mathbf{3 6 . 7} \%$ & $18.9 \%$ & $25.1 \%$ \\
Espectáculos & $20.4 \%$ & $14.8 \%$ & $20.5 \%$ & $21.5 \%$ \\
Servicio & $11.2 \%$ & $8.6 \%$ & $9.3 \%$ & $10.2 \%$ \\
Deportes & $2.1 \%$ & $1.6 \%$ & $4.4 \%$ & $5.1 \%$ \\
Otros & $11.5 \%$ & $3.4 \%$ & $11.8 \%$ & $1.0 \%$ \\
\hline Total general & $100.0 \%$ & $100.0 \%$ & $100.0 \%$ & $100.0 \%$ \\
\hline
\end{tabular}

Tampoco requiere gran argumentación la necesidad de canales regionales de TVN con programación segmentada localmente; la identidad local y la constitución de capacidad 
regional de dirigencia político-social se beneficiarían grandemente con una señal segmentada en TVN.

El interés de la audiencia por la información entregada en TV abierta se puede advertir en la primera sintonía otrogada el año 2009 a los programas de Información en TV.

El año 2009 puede ser denominado como elaño de la recepción de información, ya que la audiencia duplica el porcentaje de sintonía (36,7\%) en relación al 17,9\% de emisiones informativas, y se constituye en la categoría con primera sintonía para las audiencias. La hipótesis es que las elecciones parlamentarias y de Presidente de la República, realizadas en diciembre de 2009, atrajeron el interés de las audiencias hacia la información. El género de los informativos es predominante en la sintonía de las audiencias, y alcanza el 21,7\% del total de la sintonía a todos los programas emitidos en TV abierta. El análisis por canal (tabla 1.9; informe Obitel.cl) muestra que La Red y Telecanal, canales de propiedad extranjera, se alejan del perfil informativo de la TV chilena: predomina la ficción extranjera y espectáculos, y declina la presencia de información.

El canal de cable 24 Horas de TVN ha estado permanentemente entre los top ten del cable, disputando estrechamente la prelación con CNN Chile: ambos con 0,03 puntos de rating en el primer semestre del 2010 (Time Ibope). La sintonía a ambos canales segmentados en contenidos informativos es otra señaldelinterés de la audiencia en la información.

La tecnología digital permite ahora que la señal de cable 24 Horas de TVN pueda ser emitida en TV abierta para satisfacer elinterés de la audiencia por información, y así obtener más cobertura masiva, entregando más diversidad en géneros de información, y de modo continuado durante todo el día. La programación con diversos géneros informativos puede ayudar a mejorar la calidad en la información televisiva de la política y una mejoría en la percepción ciudadana acerca de la actividad política.

Tal canal abierto informativo de TVN, ofrece también la oportunidad para rediseñar los canales de cable del Parlamento, los cuales tienen un alto costo e ínfima sintonía, no cumpliendo los objetivos de prestigiar la actividad parlamentaria ni ampliar la deliberación ciudadana. Al incorporar la actividad parlamentaria a un canal especializado en información es posible tener más audiencia, mayor visibilidad, y prestigiar esa actividad. El dinero invertido por el Parlamento en sus canales de cable debería ser traspasado a TVN para costear un conjunto definido de programas, como por ejemplo, entrevistas regulares a los Presidentes de ambas cámaras, debates entre los jefes de bancadas, debates con los integrantes de las comisiones, debates con dirigentes sociales. Estos formatos de debate plural en pantalla acerca del trabajo del Parlamento son más atractivos para la audiencia que un boletín burocrático semanal con actividades parlamentarias; existen formatos convocantes de audiencia.

Así pues, la tecnología digital posibilita que TVN - el canal público chileno - asuma una misión de servicio público más compleja y amplia, cual es procurar más diversidad programática para el público, y con ello más calidad en su oferta a la audiencia.

Al igual que la reforma de TVN en 1992 puso un estándar informativo pluraly masivo -que se expandió a todo el sistema chileno- una regulación legal para establecer la operación 
multicanaly segmentada en TVN puede colocar un nuevo estándar de variedad a la industria chilena, con una oferta de mayor calidad para la audiencia.

\section{La reconversión industrial}

Desde que se introdujo la TV privada y mayor competencia entre canales, lentamente los nuevos canales han ido implementando un nuevo modelo de industria televisiva con una provisión de programas producidos externamente a los canales; tal proceso supone ensayo con productoras independientes y un decantamiento de las empresas más exitosas.

El análisis económico del sistema televisivo chileno a través de los balances públicos anuales está mostrando en los últimos años que si bien TVN y Canal 13, los canales más antiguos, duplican a los siguientes canales en los ingresos publicitarios, sus costos también duplican los costos de los demás canales, y sus ganancias son la mitad o la tercera parte de los canales privados.

Tal situación es insostenible en el mediano y largo plazo, ya que Canal 13 y TVN substantivamente dependen de sus propias ganancias anuales para sus procesos de renovación tecnológica y física, y para la innovación programática; el Estado chileno y la Universidad Católica, "dueños" de esos canales, tienen graves restricciones para realizar nuevas inversiones, capitalizar, o absorber pérdidas operacionales, en comparación con los canales privados; la venta reciente de Canal 13 al grupo Luksic es una prueba de ello.

Es altamente probable que los cambios que serán introducidos por la tecnología digital van acelerar la diferenciación de ambos modelos industriales, y van a presionar por la reconversión industrial; reconversión resistida activamente por los sindicatos de los canales, que demuestran una comprensible suspicacia en el corto plazo pero finalmente ceguera estratégica. Tampoco es un caso excepcional; existen canales públicos latinoamericanos cuyos sindicatos se resisten a reformas tendientes a la profesionalización.

En este sentido, TVN requiere convertirse en una empresa audiovisual multimedial (no solo televisiva) con mucha flexibilidad para entregar servicios a las audiencias y para buscar el volumen de financiamiento necesario. La BBC, por ejemplo, ha podido mantener relativamente bajo el canon por tenencia de televisor, a través de la exitosa política de exportación, con unas 40.000 horas anuales de programas y series televisivas.

Una ley para TVN no podría dictar disposiciones precisas atingentes a este nuevo escenario, ya que es muy imprevisible. Pero ciertamente debe prever un escenario general dinámico y volátil, y dotar a la empresa de la flexibilidad y agilidad necesarias para adaptarse a los nuevos contextos.

\section{Calidad en la Dirección Superior de TVN}

La ley de reforma de TVN del año 1992 instituyó un Directorio como garante y ejecutor de la misión de informar plural y balanceadamente; a juzgar por los resultados obtenidos, tal 
Directorio ha funcionado substantivamente bien, con la excepción de períodos en donde su composición conspiró contra la buena marcha del canal'.

Por tanto el tema de garantizar la calidad del Directorio de TVN es clave para su buen desempeño comunicacional. La ley del año 92 procuró algunos elementos claves en la calidad del Directorio, como:

\footnotetext{
${ }^{4}$ En los años 2003 y 2005, TVN baja del primer lugar en la sintonía general anual y en el Noticiario central ante su competencia más tradicional, canal 13, pero sigue manteniendo un respetable segundo lugar; la baja obedece, en parte, a una exitosa programación, por parte de canal 13, de la telenovela "Machos" (2003) y de "Brujas" (2005) en la franja inmediatamente anterior al Noticiario central. Sin embargo hay otra interpretación complementaria para esta baja; ella reflejaba una crisis menos visible: el Directorio de TVN renovado en el año 2000 para TVN fue nominado bajo otras ideas. En lugar de asignar una tarea compartida para el conjunto del Directorio, sus miembros debían tener una divergencia muy grande, al punto que algunos de ellos ya previamente habían manifestado que "odiaban" la TV, o habían expresado su discrepancia con algunos rasgos esenciales que la ley asignaba a TVN, como el autofinanciamiento, la importante autonomía política del Gobierno, e incluso el pluralismo informativo (calificado de noción engañosa), y añorando funciones "iluministas" en política y alta cultura. El Directorio, pues, no tenía una misión directiva básica y compartida hacia la cual dirigir al canal. En esa condición, más que dirigir buscando lo óptimo hacia una meta, el Directorio se desgastó en manejar las profundas discrepancias internas en arreglos posibles; evitar la ingobernabilidad se transformó en la tarea primordial, en lugar de actuar hacia metas positivas. Quienes designaron ese Directorio confundieron la función directiva con la función deliberativa, demostrando su escasa experiencia justamente en dirección y administración de empresas de TV. En toda empresa, un Directorio exitoso está constituido por un equipo cohesionado, con confianza mutua, optimistas acerca de una misión compartida y con una actitud creativa hacia metas de logro. Ese Directorio de TVN, internamente conflictivo y confundido introdujo una profunda inestabilidad en la empresa, la cual se manifestó también en una inseguridad desestimulante del ánimo creativo. En otros canales públicos de TV, e incluso privados, la función opinativa se ha confiado a Consejos consultivos alrededor del Directorio, pero sin interferencia con una dirección ejecutiva y dinámica, que discute sobre los medios pues los fines están ya establecidos y aceptados. Lo sorprendente en esa crisis de TVN es que ella fue menos un problema de desconfianza en la información por parte de la audiencia o de mal manejo ejecutivo, sino de un Directorio mal constituido. Eso señala lo delicado y complejo de la designación y constitución interna del Directorio de un canal Público. Ante la crisis de ingobernabilidad en TVN, en enero de 2004, Pablo Piñera renuncia a su cargo de Director Ejecutivo. El audaz paso de Piñera impide que sea destituido por el Directorio y convertido en "chivo expiatorio", pero también obligó inescapablemente a situar la crisis de ingobernabilidad en el propio Directorio. Después de la renuncia de Piñera, la consciencia de la deficiencia del Directorio, llevó al Presidente del Directorio a negociar la renuncia conjunta de todos sus miembros, para posibilitar una nueva propuesta de nominación por parte del Presidente de la República al Senado. En marzo del 2004, en acuerdo con el Senado se renovó por completo el Directorio del canal, y por designación del Presidente Lagos asumió como Presidente del Directorio Carlos Mladinic, ex ministro Secretario General de Gobierno, y en ese momento Presidente de Sistema de Empresas Públicas (SEP). El nuevo Directorio estaba conformado por personas que tenían diferentes posiciones políticas, pero compartían la misión fundamental de TVN. En julio de 2004, el Directorio de TVN nombró como Director Ejecutivo del canal al ingeniero Daniel Fernández, que en ese momento se desempeñaba como gerente general de la ENAP y que además había sido Presidente de la empresa Metro. Tanto Mladinic como Fernández generaban consenso en el mundo político, y eran considerados eficientes administradores públicos. La crisis de canal 13 de la PUC y su reciente venta (agosto de 2010), en mi opinión está profundamente ligada a una mala calidad de Dirección Superior; canal 13 nunca logró dar el paso desde una dirección ejecutiva unipersonal hacia constituir un Directorio colegiado, profesional, y responsable.
} 
- El Directorio es la autoridad superior del canal; el Directorio es el mecanismo jurídico que permite la autonomía política del Gobierno;

- El Directorio es nominado por dos poderes públicos del Estado;

- ElDirectorio transforma jurídicamente a TVN en una estación pública del Estado;

- El Directorio debe integrar internamente las mayorías y minorías políticas representadas en el Senado; esto es, es un Directorio plural constituido por personas con diferentes sensibilidades político-culturales, pero de mutua confianza pública;

- El Directorio designa por mayorías calificadas (alta confianza compartida) las autoridades superiores de la empresa;

- El Directorio es inamovible y con duración de 8 años, esto permite fortalecer la gobernabilidad empresarialy la estabilidad industrial de TVN;

- El Directorio debe garantizar una información televisiva concebida como un bien social, que representa las legítimas diferencias político-culturales significativas en el país, en lugar de transmitir propaganda política y asesinar simbólicamente al adversario;

- El Directorio de TVN garantiza una misión comunicacional de Estado, que trasciende a los Gobiernos. ${ }^{5}$

La ley del año 1992, en concordancia con la misión esencial de TVN, releva el pluralismo político en el Directorio del canal; la experiencia nacional e internacional señala que mejorar la calidad delDirectorio en canales de TV requiere reforzar otras condiciones coalescentes:

- debe ser un grupo pequeño para tomar decisiones rápidas y oportunas en un ambiente industrial altamente dinámico y competitivo;

- debe ser un grupo cohesionado que debe compartir la misión fundamental de la empresa;

- debe tener confianza interna mutua en un ambiente de legítima diversidad;

- sus miembros deben pertenecer a la nueva cultura audiovisual;

- deben tener capacidad de gestión empresarial-industrial ${ }^{6}$;

- el Directorio debe dar cuenta pública anualmente de su gestión;

\footnotetext{
${ }^{5}$ La ley de reforma de TVN también incluyó cambios administrativos para incentivar una gestión ágil y eficiente, y así competir con las demás estaciones privadas en el nuevo contexto televisivo. Dentro de estas reformas está la obligación de entregar información económica periódica pública (FECU) y un balance público anual.

${ }^{6}$ Estas dos condiciones de apreciar la cultura audiovisual - en lugar de representar a la alta cultura lecto-escrita - y de tener capacidad de gestión empresarial-industrial han sido tradicionalmente desvalorizadas en algunos sectores dirigentes; más bien ha existido el prejuicio que los representantes de la alta cultura podrían "redimir" y "elevar" a la cultura audiovisual; el aspecto de la calidad en la gestión industrial-empresarial es especialmente incomprendido en algunos sectores políticos.
} 
- la complejidad actual del escenario televisivo requiere que la empresa TVN tenga un Directorio que dedique más horas rentadas a su tarea.

Cualquier nueva reforma de TVN debe tomar en cuenta que potenciar la calidad del Directorio es clave para incrementar la calidad televisiva.

Sin embargo, el Directorio de 7 miembros de TVN ha sido criticado por ser reducido y no representar todos los matices político-culturales de la sociedad chilena. Los directorios de empresas siempre son reducidos: 7, 9 u 11 miembros como máximo. La opción reducida señala que la Reforma de TVN buscó un cuerpo con efectiva capacidad directiva; un cuerpo colegiado más amplio es en realidad un Consejo Asesor más opinativo que directivoejecutivo; las evaluaciones de los Consejos establecidos por la ley de 1970 para la TV universitaria chilena concluían en desaconsejar consejos amplios como directorio para una empresa televisiva, ya que una empresa requiere una toma de decisiones con rapidez, agilidad y efectividad (Fuenzalida, 1985). Sin embargo esa opción directiva pequeña (y absolutamente adecuada para el buen gobierno empresarial), necesita ser complementada por consejos consultivos y opinativos alrededor del mismo Directorio, a objeto de ampliar la escucha plural de voces ciudadanas; tales consejos consultivos deberían ser aún más necesarios alrededor de las señales segmentadas, que requieren más variedad de puntos de vistas con información especializada.

El siguiente esquema permite graficar, de modo general, una organización eficaz tanto en la dirección empresarial como en la participación de la ciudadanía; es un esquema general para una articulación bastante difícil de lograr, como lo muestran las evaluaciones mencionadas.

Figura 1. Propuesta de organización de TVN (fuente: elaboración propia)

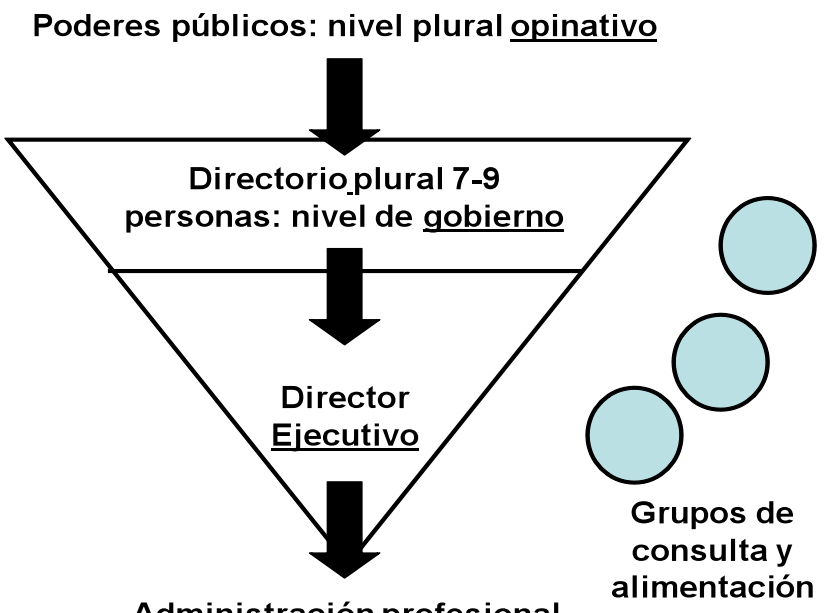

Administración profesional 


\section{Calidad en la regulación general legal}

Según la misma encuesta del CNTV en el año 2008, desde un punto de vista comparativo, la audiencia opina -otorgándole una segundo lugar (43,1\%)- que una mayor regulación estatal podría contribuir a mejorar la $T V$ abierta. Interpretamos esa opinión como una aspiración a que elCNTV tenga más capacidad legal de orientar positivamente a la TV chilena en su toma de decisiones para beneficio y satisfacción de las audiencias.

Como reacción a la dictadura, en la ley de 1992 se dispuso que el CNTV en democracia fuese muy respetuoso de la libertad de programar en las estaciones de TV (Brunner y Catalán, 1995). Las opiniones mencionadas parecen evaluar esas disposiciones como excesivamente permisivas, y demandan mayor capacidad de orientación hacia metas programáticas. Esta es una discusión reguladora delicada, ya que se puede caer en prohibiciones populistas, en protecciones corporativas, en ingenuidades infactibles, y en modas efímeras. Personalmente creo que las obligaciones programáticas a los canales deberían ser tomadas por grandes mayorías calificadas en el CNTV, que demuestren amplio consenso, y en base a sólida información que señale alta probabilidad de sus logros. Esas obligaciones deberían ser evaluadas anualmente por el mismo CNTV para verificar la realización de sus metas, a fin de perfeccionarlas o derogarlas.

El CNTV ya ha realizado un valioso aporte positivo a la TV chilena en un doble campo: estudios constantes acerca del medio televisivo (como el antes mencionado), y el concurso económico anual Fondo CNTV para incentivar la innovación en programas televisivos. Este fondo es muy realista ya que atiende a la cara industrial de la TV: aporta un dinero inicial de riesgo a programas televisivos innovadores que los canales se comprometen a emitir, pero que sin el apoyo del CNTV no se habrían producido; los aportes del Fondo CNTV han sido importantes para el desarrollo de programas innovadores; beneficia a todos los canales y a productores independientes en asociación con los canales; el éxito del Fondo ha conducido a aumentarlo progresivamente año tras año. El Fondo del CNTV por concurso se ha demostrado como un efectivo mecanismo asignador de dinero público en la TV: promoviendo programas con éxito de público en pantalla, con mecanismos evaluativos más transparentes, y un control de ejecución que inhibe la corrupción y la malversación.

Con la ampliación digital, el Fondo del CNTV para fomentar la calidad de la TV chilena tendrá que readecuarse hacia montos substantivamente más considerables, y contemplar nuevas modalidades de asignación -como fortalecer grandes bloques temáticos de programación, en lugar de simplemente programas aislados, e incluso incentivar la creación de canales segmentados, como un canal infantil chileno (y latinoamericano) en TV abierta. Esto es, en ambiente digital la calidad y la satisfacción de la audiencia ya no podrán descansar solamente en el canal público, ni en el fomento de algunos programas aislados, ni en meras regulaciones -positivas o prohibitivas- sino en la vigorizada capacidad económica del CNTV para que toda la industria televisiva pueda contribuir con mayor diversidad programática.

Sin embargo, esta acción del CNTV se focaliza más bien en el polo de la producción, en donde la ayuda se focaliza en la producción y acceso a la programación. Existen muchas preguntas y debates pendientes, como ¿debería el CNTV actuar imponiendo ciertas 
obligaciones en el polo de la emisión, como cuotas de determinados programas informativos, infantiles, ficcionales, de origen nacional, etc.? Si bien el CNTV ha dispuesto de un fondo económico creciente para incentivar la creación televisiva innovadora, la emisión efectiva de muchos de esos programas por parte de los canales ha demostrado bastante incumplimiento ${ }^{7}$. Por otra parte, el CNTV se ha movido en un horizonte nacional, y hoy se acepta que sin exportación del audiovisual, la producción tendrá enormes dificultades para sustentarse en una pequeña audiencia nacional; ¿cómo podría el CNTV impulsar la exportación de producciones televisivas?

También el nuevo escenario competitivo apunta a que otros fondos públicos, especialmente los manejados por CORFO, deben apoyar substantivamente la distribución y exportación de obras audiovisuales; y no solamente a creadores individuales sino a empresas audiovisuales exportadoras.

\section{Consideraciones finales}

La digitalización y la privatización en la TV chilena plantean ciertamente un nuevo escenario en donde las dudas e incertidumbres son más abundantes que las seguridades. Ello hace aconsejable una legislación muy general donde se prevea la delegación de decisiones particulares a un organismo colegiado más rápido y flexible, como el CNTV. Aquí se ha querido plantear algunos cambios y oportunidades que aparecen más probables, como la necesidad de responder a la insatisfacción de la audiencia con diversidad programática; la tecnología digital es una posibilidad cierta de segmentación temática en la programación de TVN, y así podría contribuir a establecer un nuevo Standard de calidad para el sistema televisivo chileno. Tal mejoría en calidad en el servicio televisivo de TVN requiere también perfeccionar el Directorio del canal.

\footnotetext{
${ }^{7}$ No podemos sino enunciar este aspecto, el cual es muy amplio y complejo para ser desarrollado aquí. Si hay obligaciones, ¿ellas serán válidas para todos los múltiples canales digitales o solo para algunos de ellos?; ¿ es aceptable que los canales de propiedad extranjera tengan tan bajo porcentaje de información y ninguna producción ficcional nacional, según datos de Obitel (Obitel.cl)?; ¿o las obligaciones serán diferenciadas y con cuáles criterios?; ¿qué significa calidad para pequeños canales comunitarios?; ante la multiplicidad de canales, ¿cómo controlar las emisiones?; ¿con cuales instrumentos evaluar la eficiencia de las regulaciones para perfeccionarlas o derogarlas?. La calidad de la TV británica ha sido atribuida a una competencia entre la BBC y las emisoras privadas al interior de un marco regulatorio con exigencias comunes. Por otra parte, las evaluaciones de la política de cuotas de ficción nacional y europea en la TV de la Unión Europea muestran que no se ha logrado fortalecer plenamente la industria nacional de ficción audiovisual - como era el objetivo - justamente porque la política se focalizó en la producción, y descuidó la distribución y circulación internacionales (Bustamante, 2004; Candeloro y Richeri, 2004).
} 


\section{Referencias}

Brunner, J.J.; Catalán, C. (1995) Televisión, Libertad, Mercado y Moral Santiago de Chile: Los Andes.

Bustamante, E. (2004) "El audiovisual europeo a la hora de la diversidad". Diálogos de la Comunicación $\mathrm{N}^{\circ} 70$. pp. 2437.

Consejo Nacional de Televisión (2008) Sexta Encuesta Nacional de TV. Televisión abierta: Niveles de Satisfacción y Percepción de Calidad. Santiago.

Candeloro, J.P.; Richeri, G. (2004) "La fiction televisiva nel mercato unico europeo". Studies in Communication Sciences. Vol. 4 № 1.

Fuenzalida, V. (2008) "Cambios en la relación de los Niños con la Televisión”. Comunicar Vol. 15 No 30. pp. 49-54.

(1985) Democratización de la Televisión chilena. Santiago: CPU.

Iyengar S.; Hahn, K.S. (2009) "Evidence of Selectivity in Media Use". Journal of Communication. Vol. 59 № 1. pp. 19-39.

Universidad Diego Portales (2007) Tercera Encuesta Nacional de Opinión Pública. Santiago de Chile. 